\title{
Correlation between TXNRD1/HO-1 expression and response to neoadjuvant chemoradiation therapy in patients with esophageal squamous cell carcinoma
}

\author{
Ryujiro Akaishi $^{1,2}$. Fumiyoshi Fujishima ${ }^{2}$. Hirotaka Ishida ${ }^{1}$ Junichi Tsunokake ${ }^{1,2} \cdot$ Takuro Yamauchi $^{1,2}$. \\ Yusuke Gokon ${ }^{1}$. Shunsuke Ueki ${ }^{1} \cdot$ Toshiaki Fukutomi $^{1} \cdot$ Hiroshi Okamoto ${ }^{1} \cdot$ Kai Takaya ${ }^{1}$. Chiaki Sato ${ }^{1}$. \\ Yusuke Taniyama ${ }^{1} \cdot$ Tomohiro Nakamura $^{3} \cdot$ Naoki Nakaya $^{4} \cdot$ Takashi Kamei $^{1} \cdot$ Hironobu Sasano $^{2}$
}

Received: 27 January 2021 / Accepted: 22 December 2021 / Published online: 8 January 2022

(c) The Author(s) 2022

\begin{abstract}
Background Thioredoxin reductase 1 (TXNRD1) and heme oxygenase-1 (HO-1) are both involved in the nuclear factor erythroid 2-related factor 2 (Nrf2) pathway and play key roles in antioxidant responses. In patients with esophageal squamous cell carcinoma (ESCC), the correlation between the expression of these two proteins and the therapeutic response to neoadjuvant chemoradiation therapy (NACRT), as well as the difference in their expression after chemoradiotherapy, remains unknown. Methods Proteins involved in the Nrf2 pathway were immunolocalized in carcinoma cells in ESCC patients on NACRT with 5-fluorouracil and cisplatin, followed by esophagectomy. The 8-hydroxydeoxyguanosine (8-OHdG) levels were used to quantify reactive oxygen species. The changes in immunoreactivity before and after NACRT $(\Delta)$ were assessed.

Results Tumor reduction following NACRT was significantly attenuated in pre-therapeutic biopsy specimens associated with high HO-1 status. TXNRD1 $\Delta$, HO-1 $1 \Delta$, and $8-\mathrm{OHdG} \Delta$ were significantly different in the ineffective and effective groups. The overall survival was significantly lower in high Nrf2 and TXNRD1 groups. In addition, high TXNRD1 expression was an independent prognostic factor in the multivariate analysis of overall survival.

Conclusions The study findings indicate that HO-1 status in pre-therapeutic biopsy specimens could predict response to NACRT, and TXNRD1 status could predict overall survival of ESCC patients.
\end{abstract}

Keywords Thioredoxin reductase $1 \cdot$ Heme oxygenase-1 $\cdot$ Esophageal squamous cell carcinoma $\cdot$ Neoadjuvant therapy $\cdot$ Chemoradiotherapy

Fumiyoshi Fujishima

ffujishima@patholo2.med.tohoku.ac.jp

1 Department of Surgery, Tohoku University Graduate School of Medicine, Sendai, Japan

2 Department of Pathology, Tohoku University Hospital, Sendai, Japan

3 Department of Health Record Informatics Information Security, Tohoku Medical Megabank Organization, Tohoku University, Sendai, Japan

4 Department of Preventive Medicine and Epidemiology, Tohoku Medical Megabank Organization, Tohoku University, Sendai, Japan

\section{Introduction}

Esophageal squamous cell carcinoma (ESCC) represents the majority of esophageal cancer cases in Japan and Asia [1]. Neoadjuvant chemotherapy (NAC) followed by surgical resection with lymph node dissection is commonly used to treat locally advanced ESCC [2]. However, strategies for treating patients with resistance to NAC are yet to be established $[3,4]$. Clinical trials on neoadjuvant chemoradiation therapy (NACRT) using 5-fluorouracil/cisplatin with concurrent radiation therapy are underway for locally advanced ESCC [5-7] and may provide novel insights into optimal therapeutic approaches.

Reactive oxygen species (ROS) are known to mediate chemotherapy- or radiotherapy-induced damages to cancer cells. Nuclear factor erythroid 2-related factor 2 (Nrf2) is a transcription factor involved in the regulation 
of antioxidant protein expression in cells [8]. Nrf2 expression is enhanced in ESCC, resulting in the development of resistance to chemotherapy and radiotherapy [9]. Nrf2 also promotes the expression of antioxidant enzymes, including thioredoxin reductase 1 (TXNRD1) [10] and heme oxygenase-1 (HO-1) [11], which increases resistance to oxidative stress. TXNRD1 is one of the key enzymes that defend cancer cells against oxidative stress [10] and promotes cell proliferation and viability [12]. HO-1 is also an Nrf2 target, and its involvement in cell proliferation and development in cancer has been extensively studied $[13,14]$.

Low Nrf2 expression in pre-NACRT biopsy specimens was reported to be correlated with a favorable response to NACRT in ESCC patients [15]. However, the correlation between TXNRD1/HO-1 expression and response to NACRT has remained unknown. In addition, the prognostic significance of the proteins involved in these antioxidant pathways in human malignancies is yet to be established.

Therefore, in this study, we aimed to investigate the followings in ESCC patients: (1) predict NACRT efficacy and clinical outcomes/prognosis according to the status of TXNRD1/HO-1 in pre-NACRT endoscopic biopsy specimens and (2) examine the correlation between NACRT resistance and the difference of antioxidant protein expression in pre- and post-NACRT specimens.

\section{Materials and methods}

\section{Patients and tumor specimens}

The study enrolled 69 patients diagnosed with ESCC who underwent NACRT followed by thoracoscopic esophagectomy with regional lymph node dissection between 2011 and 2015 at Tohoku University Hospital, Sendai, Japan. Seventeen cases in which pre-therapeutic biopsy was not performed in our institution were tentatively excluded in this study. The tissue specimens available for comparison before and after NACRT were 39 cases (Supplementary Fig. 1). The criteria for therapeutic response to NACRT were tentatively determined as in Supplementary Table 1 [16]. Grade 0 or 1 response was interpreted as "ineffective," whereas a Grade 2 or 3 response was considered "effective" [15].

TNM (tumor, nodes, and metastasis) staging was performed according to the guidelines defined in the eighth edition of the American Joint Committee on Cancer/Union for International Cancer Control [17]. The overall survival (OS) and recurrence-free survival (RFS) in patients were determined from the day NACRT commenced until death and recurrence, respectively, or based on the last follow-up.

The study protocol was approved by the Ethics Committee of the Tohoku University School of Medicine (Accession No. 2020-1-87), and informed consent was obtained from all participants prior to surgery.

\section{Neoadjuvant chemoradiation therapy}

Chemotherapy was administered in conjunction with continuous intravenous infusion of 5 -fluorouracil $\left(400 \mathrm{mg} / \mathrm{m}^{2} /\right.$ day) for over $24 \mathrm{~h}$ on days $1-5$ and $8-12$ and with cisplatin $\left(40 \mathrm{mg} / \mathrm{m}^{2}\right)$ infusion for $2 \mathrm{~h}$ on days 1 and 8 . Concurrent radiotherapy (total of $30 \mathrm{~Gy}$ in 15 fractions over 3 weeks) was performed.

\section{Immunohistochemistry}

Immunohistochemical analyses were performed on formalin-fixed paraffin-embedded 4-micron-thick tissue sections. The immunohistochemical procedures are summarized in Supplementary Table 2. ROS levels in the tumor cells were evaluated using 8-hydroxydeoxyguanosine (8-OHdG) [18].

Each stained section was independently evaluated at the hot spots using $200 \times$ magnification by two authors (RA and FF). Immunoreactivities of Nrf2 in the nuclei, TXNRD1, HO-1, and 8-OHdG in the cytoplasm were assessed semi-quantitatively using modified $\mathrm{H}$-scores and by calculating the percentage of immunostained tumor cells multiplied by the relative immunointensity $(0$, negative; 1, weak; 2, moderate; 3, marked) [19]. Labeling index was applied for nuclear Ki-67 immunoreactivity evaluation [20]. The optimal cutoff values for the response of the patients were determined for pre-NACRT biopsies using the receiver operating characteristic curve (Supplementary Table 3) [19]. Differences in H-score and Ki-67 labeling index ( $\Delta$ : post-NACRT - pre-NACRT values) were calculated.

\section{Statistical analysis}

JMP® Pro version 14.2.0 (SAS Institute, Inc., Cary, NC, USA) was used for statistical analyses. Pearson's 
chi-square tests, Fisher's exact tests, Student's $t$-tests, and Wilcoxon's rank-sum tests were applied as appropriate. OS and RFS rates were investigated using the Kaplan-Meier method and compared using log-rank tests. The Cox proportional hazards model was used for univariate and multivariate analyses. Multivariate logistic regression analyses were also conducted. Variance inflation factor (VIF) among explanatory variables was calculated for each multivariate analysis. We confirmed that there was no multicollinearity in each multivariate analysis and VIF for each variable was $\leq 5 . P<0.05$ was considered significant.

\section{Results}

\section{Clinicopathological features of ESCC patients}

The clinical characteristics of 52 cases are summarized in Table 1. Among these, the samples of 25 patients were tentatively classified as ineffective (Grade 0 or 1 ), and the remaining 27 patients exhibited a Grade 2 or 3

Table 1 Clinical features of the patients

\begin{tabular}{|c|c|c|c|c|}
\hline \multirow[t]{2}{*}{ Clinical features } & \multirow[t]{2}{*}{$N$} & $\begin{array}{l}\text { Ineffective } \\
\text { (Grade } 0-1 b \text { ) }\end{array}$ & $\begin{array}{l}\text { Effective } \\
\text { (Grade 2-3) }\end{array}$ & \multirow[t]{2}{*}{$P$ value } \\
\hline & & 25 & 27 & \\
\hline Age $\geq 65$ & 30 & 15 & 15 & 0.746 \\
\hline Age $<65$ & 22 & 10 & 12 & \\
\hline Male & 41 & 18 & 23 & 0.317 \\
\hline Female & 11 & 7 & 4 & \\
\hline Smoker & 38 & 18 & 20 & 0.866 \\
\hline Non-smoker & 14 & 7 & 7 & \\
\hline Main location & & & & 0.488 \\
\hline Upper/Middle thoracic & 35 & 18 & 17 & \\
\hline $\begin{array}{l}\text { Lower thoracic/Esoph- } \\
\text { agogastric junction }\end{array}$ & 17 & 7 & 10 & \\
\hline $\mathrm{cT}^{\mathrm{a}}$ & & & & 0.069 \\
\hline $\mathrm{cT} 1 / 2$ & 15 & 4 & 11 & \\
\hline $\mathrm{cT} 3 / \mathrm{T} 4 \mathrm{a}$ & 37 & 21 & 16 & \\
\hline $\mathrm{cN}^{\mathrm{a}}$ & & & & 0.055 \\
\hline cNO & 13 & 3 & 10 & \\
\hline $\mathrm{cN} 1 / 2$ & 39 & 22 & 17 & \\
\hline Clinical stage $^{\mathrm{a}}$ & & & & $0.005 *$ \\
\hline Stage I/II & 23 & 6 & 17 & \\
\hline Stage III/IV & 29 & 19 & 10 & \\
\hline
\end{tabular}

*Statistical significance

a Tumor-node-metastasis (TNM) classification based on the 8th edition of the TNM classification of malignant tumors

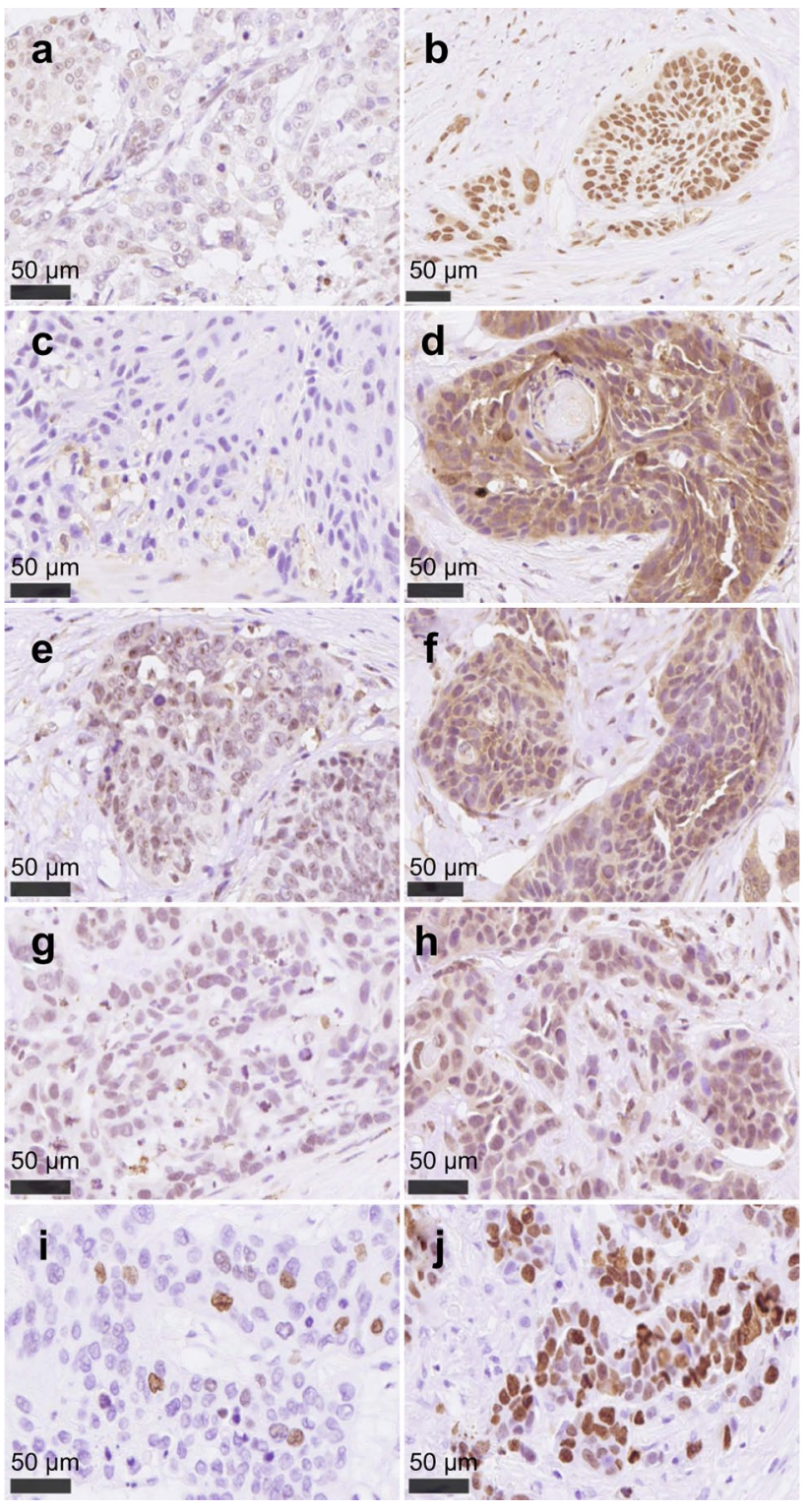

Fig. 1 Representative illustration of immunohistochemical features. a Low Nrf2 expression. b High Nrf2 expression: representative specimen depicting immunoreactivity in the nuclei of carcinoma cells. c Low TXNRD1 expression. d High TXNRD1 expression. e Low HO-1 expression. f High HO-1 expression. g Low 8-OHdG levels. h High 8-OHdG levels: representative specimen depicting immunoreactivity in the cytoplasm of carcinoma cells. $\mathbf{i}$ Low Ki-67 levels. j High Ki-67 levels: representative specimen depicting immunoreactivity in the nuclei of carcinoma cells

response to NACRT upon pathological analysis of the resected specimens. There were significant differences in the clinical (c) Stage between the ineffective and effective groups. 
Table 2 Multivariate logistic regression analysis of histological NACRT efficacy in Pre-NACRT biopsy specimens

\begin{tabular}{lllll}
\hline Variables & & Odds ratio $(95 \%$ CI $)$ & $P$ & VIF \\
\hline Age & <65 vs. 65 $\leq$ & $2.711(0.341-21.536)$ & 0.345 & 1.753 \\
Gender & Male vs. Female & $0.028(0.001-0.894)$ & $0.043^{*}$ & 3.149 \\
$\mathrm{cT}^{\dagger}$ & cT1-2 vs. cT3-4 & $0.034(0.002-0.473)$ & $0.012 *$ & 2.289 \\
$\mathrm{cN}^{\dagger}$ & cN0 vs. cN1-2 & $0.102(0.012-0.889)$ & $0.039^{*}$ & 1.333 \\
Nrf2 & Low vs. High & $0.141(0.015-1.340)$ & 0.088 & 1.391 \\
TXNRD1 & Low vs. High & $0.140(0.010-1.909)$ & 0.140 & 2.617 \\
HO-1 & Low vs. High & $0.021(0.001-0.860)$ & $0.041 *$ & 2.362 \\
8-OHdG & Low vs. High & $0.991(0.969-1.015)$ & 0.462 & 1.430 \\
\hline
\end{tabular}

NACRT neoadjuvant chemoradiation therapy; VIF variance inflation factor

* Statistical significance

${ }^{\dagger}$ Tumor-node-metastasis (TNM) classification based on the 8th edition of the TNM classification of malignant tumors

\section{Expression status of antioxidant proteins and its correlation with response to NACRT}

Representative histopathological findings for Nrf2, TXNRD1, HO-1, 8-OHdG, and Ki-67 are presented in Fig. 1. A significant positive correlation was detected between Nrf2 and TXNRD1 $(P=0.001)$ and TXNRD1 and HO-1 $(P=0.025)$ (Supplementary Fig. 2). Multivariate logistic regression analysis revealed that $\operatorname{Gender}(P=0.043)$, cT $(P=0.012), \mathrm{cN}(P=0.039)$, and HO-1 $(P=0.041)$ were independent predictive factors of histological NACRT efficacy (Table 2).

\section{Correlation between the expression status of antioxidant proteins and clinical outcomes/ prognostic factors in patients}

The five-year OS rate was significantly lower in the high Nrf2 $(P=0.007)$ and TXNRD1 $(P=0.007)$ expression groups in ESCC patients (Fig. 2). No significant differences were detected in the analysis of the five-year RFS (Fig. 2). Univariate analysis revealed that the OS rate was significantly associated with high Nrf2 $(P=0.018)$ and TXNRD1 $(P=0.011)$ status (Table 3$)$. Multivariate analysis revealed that high TXNRD1 status was the only independent prognostic factor among the variables examined in this study $(P=0.049)$ (Table 3$)$. None of the variables examined was significantly associated with RFS (Table 3).

\section{Correlation between differences in expression $(\Delta)$ and NACRT resistance}

TXNRD1 $\Delta(P=0.048)$, HO-1 $\Delta(P=0.021)$, and $8-\mathrm{OHdG} \Delta$ $(P=0.048)$ were significantly different in the NACRT-ineffective groups compared to the NACRT-effective groups (Fig. 3).

\section{Discussion}

First, the results of our present study revealed that HO-1 status in pre-NACRT endoscopic biopsy specimens could predict the efficacy of NACRT in the patients. In addition, TXNRD1 status in the pre-therapeutic endoscopic biopsy specimens predicted OS of the patients examined in this study. Surgical resection without NAC or NACRT could therefore facilitate curative resection in ESCC patients, who exhibited high HO-1 status, because of the frequent ineffectiveness of neoadjuvant therapy in the patients associated with aggressive biological behavior [12, 21, 22]. However, the prognosis of curative resection without preoperative therapy is certainly poor [3], which is a limitation associated with therapeutic strategies in ESCC. Further investigation of new adjuvant therapeutic strategies is necessary.

TXNRD1 $\Delta$, HO- $1 \Delta$, and 8-OhdG $\Delta$ were significantly associated with therapeutic efficacy. The significant difference in TXNRD1 $\Delta$, HO-1 $\Delta$, and 8-OHdG $\Delta$ values between the ineffective and effective groups also suggested that the ineffective group elicited a greater antioxidant response. However, no significant differences were observed in $\operatorname{Nrf} 2 \Delta$ values between the two groups. These results indicate that patients in the NACRT-ineffective group exhibit an antioxidant response that involves a selective and stronger upregulation of TXNRD1 and HO-1 expression.

The present study has some limitations. First, ESCC is characterized by intertumoral heterogeneity; therefore, the endoscopic biopsy site could have considerably influenced antioxidant protein expression. Second, as carcinoma cells completely disappeared after NACRT in some cases, protein expression changes in these cells remained unevaluated. Third, the sample size of the study was rather small and may not have been sufficient to deny that the results of multivariate analysis are by chance. Further investigations with larger sample size are required to elucidate the clinicopathological significance of results of our present study. 
Fig. 2 Kaplan-Meier estimates of OS and RFS based on pre-NACRT expression status of biomarkers. Kaplan-Meir estimates of OS (a, b, c, d, e) and RFS (a, b, c, d, e). The fiveyear OS was significantly lower in pre-NACRT specimens with high a Nrf2 and b TXNRD1 expression. $O S$ overall survival; $R F S$ recurrence-free survival; $N A C R T$ neoadjuvant chemoradiation therapy

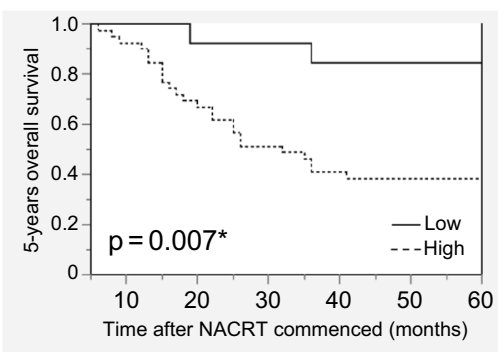

b

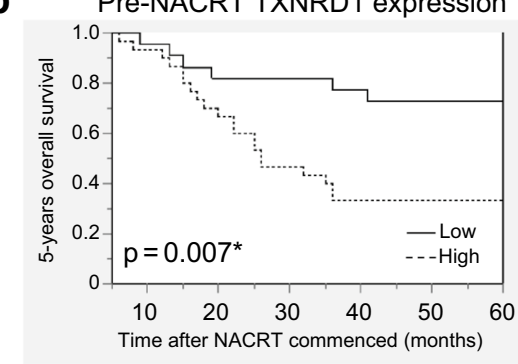

C

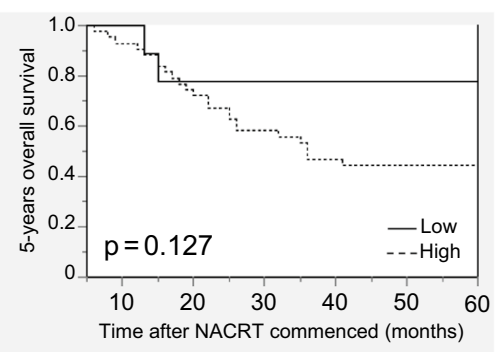

d

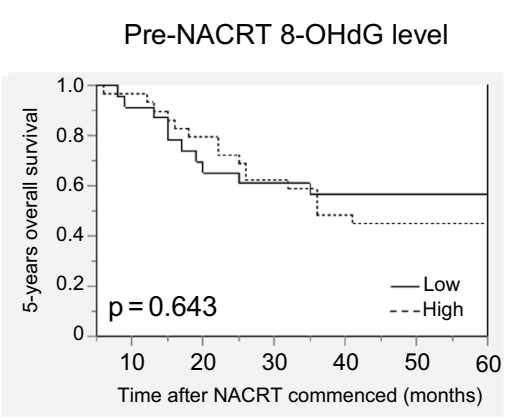

e

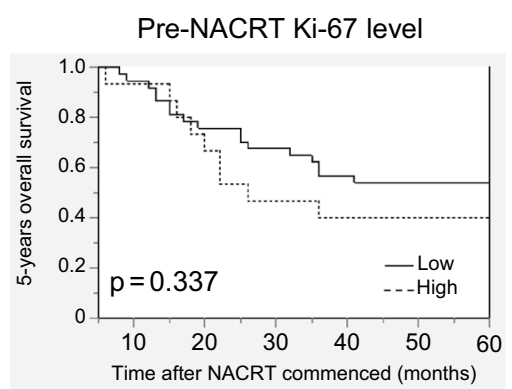

Pre-NACRT Nrf2 expression

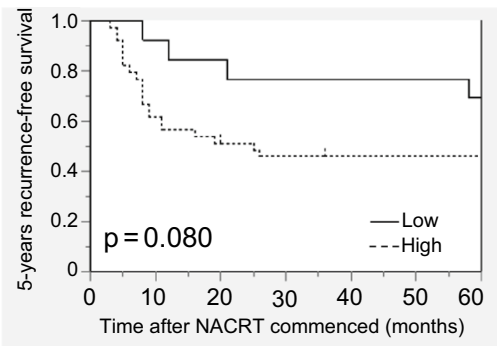

g

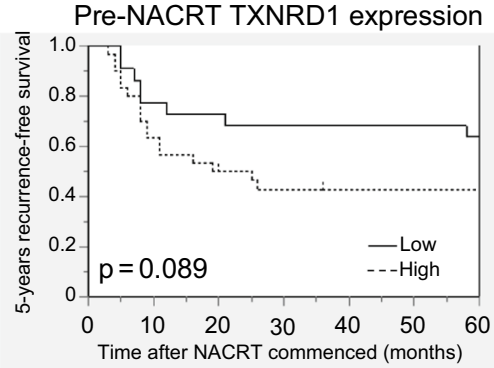

h

Pre-NACRT HO-1 expression

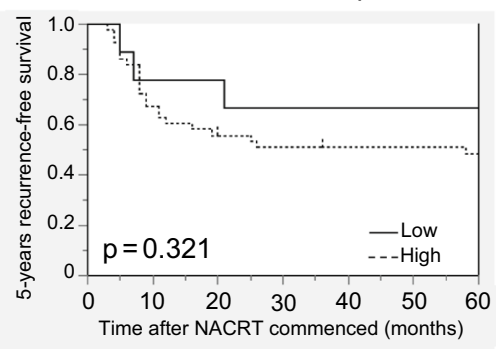

i

Pre-NACRT 8-OHdG level

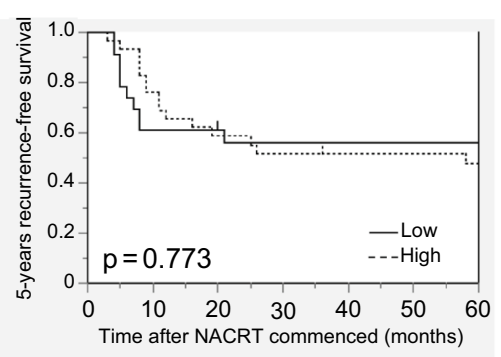

Pre-NACRT Ki-67 level

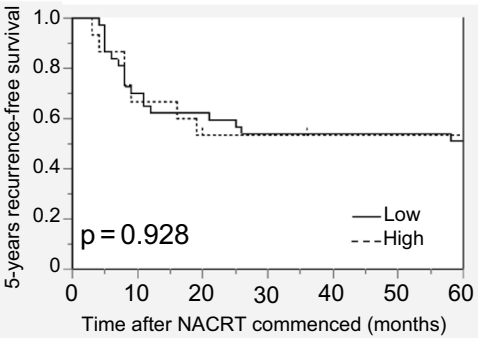


Table 3 Univariate and Multivariate analysis of 5-year OS and RFS in Pre-NACRT biopsy specimens

\begin{tabular}{|c|c|c|c|c|c|c|c|c|}
\hline \multirow[t]{2}{*}{ Variables } & & \multicolumn{2}{|c|}{ Univariate analysis (OS) } & \multicolumn{2}{|c|}{ Multivariate analysis (OS) } & \multirow[t]{2}{*}{ VIF } & \multicolumn{2}{|c|}{ Univariate analysis (RFS) } \\
\hline & & $\mathrm{HR}(95 \% \mathrm{CI})$ & $P$ & HR $(95 \% \mathrm{CI})$ & $P$ & & HR $(95 \%$ CI $)$ & $P$ \\
\hline Age & $<65$ vs. $65 \leq$ & $0.727(0.329-1.602)$ & 0.428 & $0.389(0.149-1.015)$ & 0.054 & 1.230 & $0.483(0.209-1.114)$ & 0.088 \\
\hline Gender & Male vs. Female & $0.968(0.365-2.568)$ & 0.948 & $0.326(0.559-5.735)$ & 0.326 & 1.364 & $1.324(0.531-3.301)$ & 0.547 \\
\hline $\mathrm{cT}^{\dagger}$ & cT1-2 vs. cT3 & $1.442(0.579-3.592)$ & 0.432 & $1.439(0.541-3.831)$ & 0.466 & 1.135 & $1.357(0.569-3.235)$ & 0.491 \\
\hline $\mathrm{cN}^{\dagger}$ & cN0 vs. cN1-2 & $0.901(0.379-2.145)$ & 0.814 & $0.655(0.242-1.771)$ & 0.405 & 1.140 & $1.629(0.614-4.323)$ & 0.327 \\
\hline Nrf2 & Low vs. High & $5.754(1.356-24.412)$ & $0.018^{*}$ & $2.713(0.523-14.067)$ & 0.235 & 1.705 & $2.483(0.854-7.221)$ & 0.095 \\
\hline TXNRD1 & Low vs. High & $3.280(1.309-8.218)$ & $0.011 *$ & $3.354(1.007-11.173)$ & $0.049 *$ & 1.910 & $2.010(0.872-4.635)$ & 0.101 \\
\hline HO-1 & Low vs. High & $2.893(0.683-12.255)$ & 0.149 & $4.412(0.724-26.902)$ & 0.108 & 1.456 & $1.807(0.542-6.028)$ & 0.336 \\
\hline 8-OHdG & Low vs. High & $1.203(0.545-2.651)$ & 0.647 & $0.685(0.253-1.855)$ & 0.456 & 1.468 & $1.121(0.508-2.475)$ & 0.777 \\
\hline $\mathrm{Ki}-67$ & Low vs. High & $1.477(0.657-3.321)$ & 0.345 & $0.685(0.371-2.072)$ & 0.764 & 1.301 & $0.961(0.404-2.289)$ & 0.929 \\
\hline
\end{tabular}

$O S$ overall survival; RFS recurrence-free survival; NACRT neoadjuvant chemoradiation therapy; VIF variance inflation factor

*Statistical significance

'Tumor-node-metastasis (TNM) classification based on the 8th edition of the TNM classification of malignant tumors

Fig. 3 Correlation between differences in expression $(\Delta)$ and responses to NACRT. Significant differences were observed for b TXNRD1 $\Delta(P=0.048)$, c HO-1 $\Delta(P=0.021)$, and d 8-OHdG $\Delta(P=0.048)$ between the NACRT-effective and -ineffective groups. No significant differences were observed for a $\operatorname{Nrf2} \Delta(P=0.356)$ and e Ki-67 $\Delta$ $(P=0.538)$. NACRT neoadjuvant chemoradiation therapy
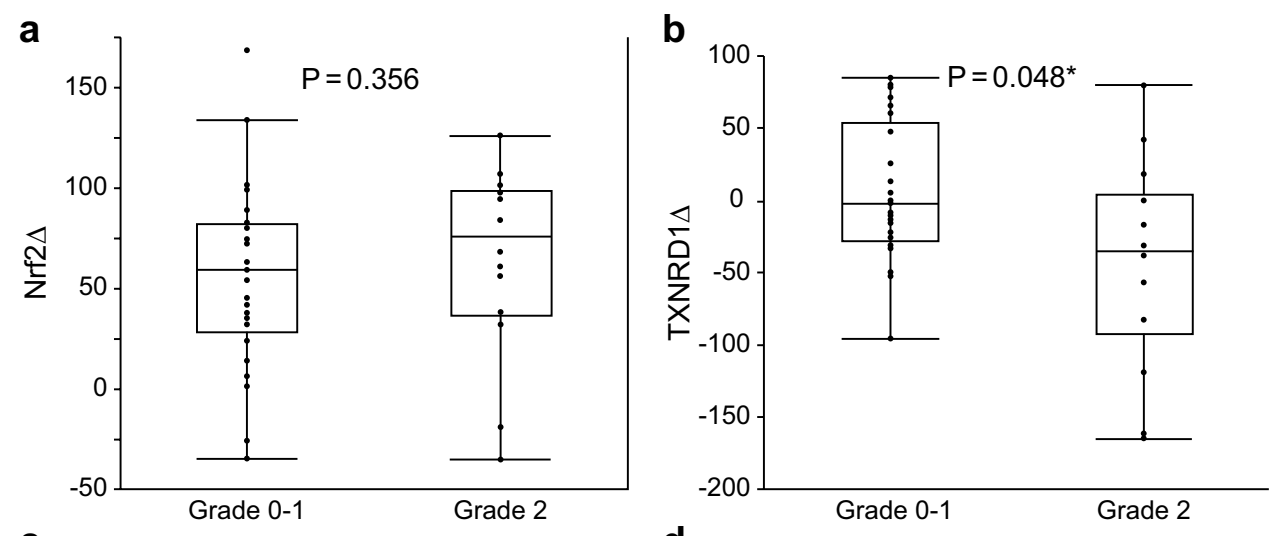

C

d
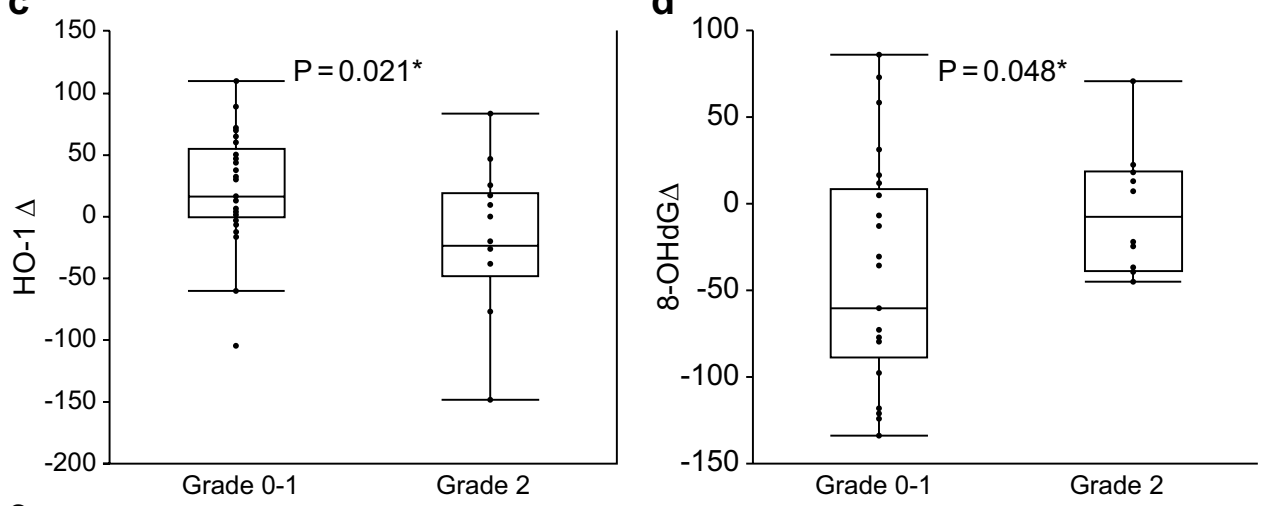

e

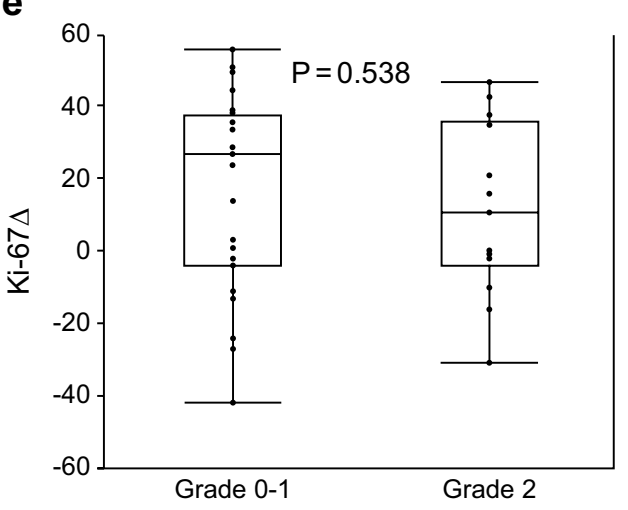




\section{Conclusions}

NACRT therapeutic efficacy and clinical outcomes in ESCC patients can be predicted by examining TXNRD1 and HO-1 expression status in carcinoma cells in pre-therapeutic endoscopic biopsy and surgically resected specimens. Further investigation could lead to the identification of potential prognostic factors for ESCC.

Supplementary Information The online version contains supplementary material available at https://doi.org/10.1007/s10388-021-00904-3.

Acknowledgements RA and FF performed immunohistochemistry. RA, FF, HI, TN, and NN performed the statistical analyses. RA, FF, HI, JT, TY, YG, SU, TF, HO, KT, CS, YT, TN, NN, TK, and HS, conceived the study, participated in its design and coordination, and helped draft the manuscript. All authors have read and approved the final manuscript. We appreciate the technical assistance provided by Ms. Yayoi Aoyama (Department of Pathology, Tohoku University Hospital, Sendai, Japan). We would like to thank Editage (www.editage. com) for English language editing.

Funding No funding or support is associated with this study.

\section{Declarations}

Ethical statement All procedures were in accordance with the ethical standards of the responsible committee on human experimentation (institutional and national) and with the Helsinki Declaration of 1964 and later versions.

Conflict of interest Authors RA, FF, HI, JT, TY, YG, SU, TF, HO, KT, CS, YT, TN, NN, TK, and HS, declare that they have no conflict of interest.

Informed consent Informed consent or substitute for it was obtained from all patients for being included in the study.

Open Access This article is licensed under a Creative Commons Attribution 4.0 International License, which permits use, sharing, adaptation, distribution and reproduction in any medium or format, as long as you give appropriate credit to the original author(s) and the source, provide a link to the Creative Commons licence, and indicate if changes were made. The images or other third party material in this article are included in the article's Creative Commons licence, unless indicated otherwise in a credit line to the material. If material is not included in the article's Creative Commons licence and your intended use is not permitted by statutory regulation or exceeds the permitted use, you will need to obtain permission directly from the copyright holder. To view a copy of this licence, visit http://creativecommons.org/licenses/by/4.0/.

\section{References}

1. Tachimori Y, Ozawa S, Numasaki $\mathrm{H}$, et al. Comprehensive registry of esophageal cancer in Japan, 2012. Esophagus. 2019;16:221-45.

2. Kitagawa Y, Uno T, Oyama T, et al. Esophageal cancer practice guidelines 2017 edited by the Japan Esophageal Society: Part 1. Esophagus. 2019;16:1-24.
3. Ando N, Kato $\mathrm{H}$, Igaki $\mathrm{H}$, et al. A randomized trial comparing postoperative adjuvant chemotherapy with cisplatin and 5-fluorouracil versus preoperative chemotherapy for localized advanced squamous cell carcinoma of the thoracic esophagus (JCOG9907). Ann Surg Oncol. 2012;19:68-74.

4. Medical Research Council Oesophageal Cancer Working Group. Surgical resection with or without preoperative chemotherapy in oesophageal cancer: a randomised controlled trial. Lancet. 2002;359:1727-33.

5. Okumura H, Uchikado Y, Omoto I, et al. The usefulness of neoadjuvant chemoradiation therapy for locally advanced esophageal cancer with multiple lymph-node metastases. Ann Surg Oncol. 2014;21:2845-9.

6. Ishida K, Ando N, Yamamoto S, et al. Phase II study of cisplatin and 5-fluorouracil with concurrent radiotherapy in advanced squamous cell carcinoma of the esophagus: a Japan Esophageal Oncology Group (JEOG)/Japan Clinical Oncology Group Trial (JCOG9516). Jpn J Clin Oncol. 2004;34:615-9.

7. Kato K, Muro K, Minashi K, et al. Gastrointestinal Oncology Study Group of the Japan Clinical Oncology Group (JCOG): Phase II study of chemoradiotherapy with 5-fluorouracil and cisplatin for Stage II-III esophageal squamous cell carcinoma: JCOG trial (JCOG 9906). Int J Radiat Oncol Biol Phys. 2011;81:684-90.

8. Motohashi H, Yamamoto M. Nrf2-Keap1 defines a physiologically important stress response mechanism. Trends Mol Med. 2004;10:549-57.

9. Shibata T, Kokubu A, Saito S, et al. NRF2 mutation confers malignant potential and resistance to chemoradiation therapy in advanced esophageal squamous cancer. Neoplasia. 2011;13:864-73.

10. Lu J, Holmgren A. The thioredoxin antioxidant system. Free Radic Biol Med. 2014;66:75-87.

11. Alam J, Stewart D, Touchard C, et al. Nrf2, a Cap'n'Collar transcription factor, regulates induction of the heme oxygenase- 1 gene. J Biol Chem. 1999;274:26071-8.

12. Arnér ES, Holmgren A. The thioredoxin system in cancer. Semin Cancer Biol. 2006;16:420-6.

13. Maines MD, Abrahamsson PA. Expression of heme oxygenase-1 (HSP32) in human prostate: normal, hyperplastic, and tumor tissue distribution. Urology. 1996;47:727-33.

14. Goodman AI, Choudhury M, da Silva JL, et al. Overexpression of the heme oxygenase gene in renal cell carcinoma. Proc Soc Exp Biol Med. 1997;214:54-61.

15. Kawasaki Y, Okumura H, Uchikado Y, et al. Nrf2 is useful for predicting the effect of chemoradiation therapy on esophageal squamous cell carcinoma. Ann Surg Oncol. 2014;21:2347-52.

16. Japan Esophageal Society. Japanese classification of esophageal cancer, 11th edition: part II and III. Esophagus. 2017;14:37-65.

17. Brierley JD, Gospodarowicz MK, Wittekind C. TNM classification of malignant tumours. 8th ed. Wiley-Blackwell; 2017.

18. Ock CY, Hong KS, Choi KS, et al. A novel approach for stressinduced gastritis based on paradoxical anti-oxidative and antiinflammatory action of exogenous 8-hydroxydeoxyguanosine. Biochem Pharmacol. 2011;81:111-22.

19. Ueki S, Fujishima F, Kumagai T, et al. GR, Sgk1, and NDRG1 in esophageal squamous cell carcinoma: their correlation with therapeutic outcome of neoadjuvant chemotherapy. BMC Cancer. 2020;20:161.

20. Kitano Y, Baba Y, Nakagawa S, et al. Nrf2 promotes oesophageal cancer cell proliferation via metabolic reprogramming and detoxification of reactive oxygen species. J Pathol. 2018;244:346-57.

21. Furfaro AL, Traverso N, Domenicotti C, et al. The Nrf2/HO-1 axis in cancer cell growth and chemoresistance. Oxid Med Cell Longev. 2016;2016:1958174.

22. Meuillet EJ, Mahadevan D, Berggren M, et al. Thioredoxin-1 binds to the $\mathrm{C} 2$ domain of PTEN inhibiting PTEN's lipid 
phosphatase activity and membrane binding: a mechanism for the functional loss of PTEN's tumor suppressor activity. Arch Biochem Biophys. 2004;429:123-33.

Publisher's Note Springer Nature remains neutral with regard to jurisdictional claims in published maps and institutional affiliations. 